\title{
ESTUDIO PETROGRÁFICO DE CERÁMICAS MAYAS, CLÁSICO TARDÍO (600-900 D.C.), CHINIKIHÁ, CHIAPAS, MÉXICO
}

\author{
PETROGRAPHYC STUDIES FROM MAYAN CERAMICS, \\ LATE CLASSIC (600-900 A.D.), CHINIKIHA, CHIAPAS, MEXICO
}

\author{
Luis G. Obando ${ }^{1 *}$, Socorro Jiménez ${ }^{2} \&$ Siegfried Kussmaul $^{1}$ \\ ${ }^{1}$ Escuela Centroamericana de Geología, Universidad de Costa Rica. \\ ${ }^{2}$ Universidad Autónoma de Yucatán (UADY) \\ *Autor para contacto: lobando@geologia.ucr.ac.cr
}

(Recibido: 22/11/2010; aceptado: 1/06/2011)

\begin{abstract}
Chinikiha is a Maya archaeology place from Classic period (250-900 a.D.); it is located in Chiapas and Tabasco States, Mexico. Chinikiha has at least 120 monumental structures, palace type and pyramidal structures. The petrography studies of 21 ceramic samples, shows: a) Siliciclastic pastes (with 45,5- 84,5\% matrix, the average is $69,19 \%$ ) and b) carbonated pastes (with $18-64 \%$ matrix, carbonate $79 \%$, the average $30,5 \%$ ). The carbonated pastes has grain support but the silicoclastic pastes has matrix support.

The earliest Mayan-ceramic pastes are carbonates compounds but later, they were changed by siliciclastic compounds. The technologyc changes probably occurred when the Mayan decided to change the carbonated pastas by siliciclastic pastes. The Chinikihá pots, are $80 \%$ made using siliciclastic pastes, with mica. The ceramic-fire temperature probably did not reach $950^{\circ} \mathrm{C}$, instead the carbonated pastes probably did not $800^{\circ} \mathrm{C}$.

If the raw material was not imported, probably the rock source coming from Eocene sandstones or lutites rocks or Miocene sandstones or both. Furthermore, the volcanic shards found in the thin sections, possible coming from volcanic soils, for instance, Chichon volcano-tuffs deposits.

Some pastes shows the diatoms fossils. The raw source, probably, come from fresh water lakes.

Key Word: Chinikihá, Maya, Late Classic, archaeology, petrography, ceramic, pottery, glass.

RESUMEN: Chinikihá, es un sitio arqueológico maya del Clásico (250-900 d.C.), ubicado en la región occidente de la zona maya, en el territorio mexicano de la cuenca media del río Usumacinta en los estados mexicanos de Chiapas y Tabasco. En el área se ubican al menos 120 estructuras, y comprende desde edificios monumentales tipo palacio hasta estructuras piramidales.

Para los estudios petrográficos de cerámicas, se analizaron 21 muestras de fragmentos. Los resultados de las secciones delgadas analizadas en Chinikihá muestran dos tendencias de componentes base: a) los componentes silicoclásticos (con 45,5 al $84,5 \%$ de matriz arcillosa con un promedio de $69,19 \%$ ) y b) el componente carbonatado con un contenido
\end{abstract}


de matriz arcillosa entre el 18 al $64 \%$, el promedio es de 30,5\% y carbonato de hasta $79 \%$. El soporte por matriz es más notorio en las pastas silicoclásticas, mientras que las pastas carbonatadas tienen a tener un soporte por grano. Se considera que las pastas cerámicas carbonatadas fueron las más tempranas, luego dichas pastas fueron sustituidas por las silicoclásticas. El cambio tecnológico y de mentalidad en el uso de cerámicas, probablemente ocurrió al sustituir las pastas carbonatas por pastas silicoclásticas. Para este análisis, el $80 \%$ de las cazuelas de Chinikihá proceden del grupo silicoclástico con micas dominantes. Las temperaturas de cocción de las cerámicas silicoclásticas probablemente no superaron $\operatorname{los} 950^{\circ} \mathrm{C}$, mientras que las cerámicas carbonatadas no pudieron alcanzar los $800^{\circ} \mathrm{C}$.

Si la materia prima para la manufactura de las cerámicas no fue importada, se infiere que el origen esta materia prima puede ser las areniscas y lutitas del Eoceno, o bien las areniscas de edad Miocena, o ambas. Además, las esquirlas de vidrio observadas, posiblemente procedieron de las tierras volcánicas, por ejemplo, las tefras del volcán Chichón.

Algunas pastas presentan fósiles de diatomeas, lo que hace pensar que probablemente la fuente de la materia prima arcillosa estaba cercana a lagos o lagunas de agua dulce.

Palabras clave: Chinikihá, Maya, Clásico Tardío, arqueología, petrografía, cerámica, vidrio.

\section{ANTECEDENTES}

Chinikihá es un sitio arqueológico localizado al norte del Estado de Chiapas, México. Teobert Maler en 1898, fue el primer explorador que reportó la existencia de Chinikihá, lapso en el cual realiza un registro somero del estado de conservación y características generales del sitio. Con ésta única referencia, Chinikihá fue incluido en el Atlas Arqueológico de 1939 y seguidamente en el mapa de la Universidad de Tulane en 1940, para después aparecer en el Atlas Arqueológico publicado en 1967. En la década de los setenta, Robert Rands visita el sitio y excava algunos pozos de sondeo como parte de la estrategia de estudios cerámicos en las Tierra Bajas Noroccidentales que han sido de manera tradicional considerada culturalmente colindante con la zona maya del Petén Central Guatemalteco (Liendo, 2007)

Finalmente, en 1993 el sitio fue visitado por Alfonso Grave Tirado, quién excavó algunas de sus estructuras del sitio y en 2003, David Stuart y un grupo de investigadores visitan Chinikihá con el fin de elaborar un reporte que hasta la fecha constituye la síntesis más completa sobre la historia del asentamiento.

Según Liendo (2007), en agosto del 2003, a través del "Proyecto Integración Política del Señorío de Palenque" se visita el sitio elaborando un plano más exacto de la distribución de los edificios que componen al sitio y mediante pruebas de pala, se excavan algunos pozos de sondeo con la intención de obtener una muestra cerámica representativa que permitiera una aproximación temporal preliminar de la ocupación del sitio. Posteriormente, durante el 2005 se efectuaron recolecciones de superficie y excavaciones de 14 pozos y algunas calas tanto en el núcleo residencial del asentamiento como en áreas cercanas al mismo. Es importante señalar que el asentamiento precolombino, se seguirá excavando durante los años futuros.

\section{Características del sitio arqueológico y trabajos previos}

Chinikihá es un sitio ubicado en la región occidente de la zona maya, en el territorio mexicano de la cuenca media del río Usumacinta, Chiapas y Tabasco (Fig. 1). Cabe indicar que este asentamiento abarca un área extensa de aproximadamente 70 hectáreas a lo largo de la carretera que une al poblado de Reforma Agraria (Chiapas) con el de Gregorio Méndez (Pénjamo, Tabasco) (Fig 1a). Sus edificios se ubican en las cimas y laderas de los cerros del lugar, así como los pequeños valles que se forman entre ellos (Liendo, 2007).

En el área se ubican al menos 120 estructuras, desde edificios de dimensiones considerables tipo palacio y estructuras piramidales, hasta pequeñas plataformas de menos de un metro de altura, 

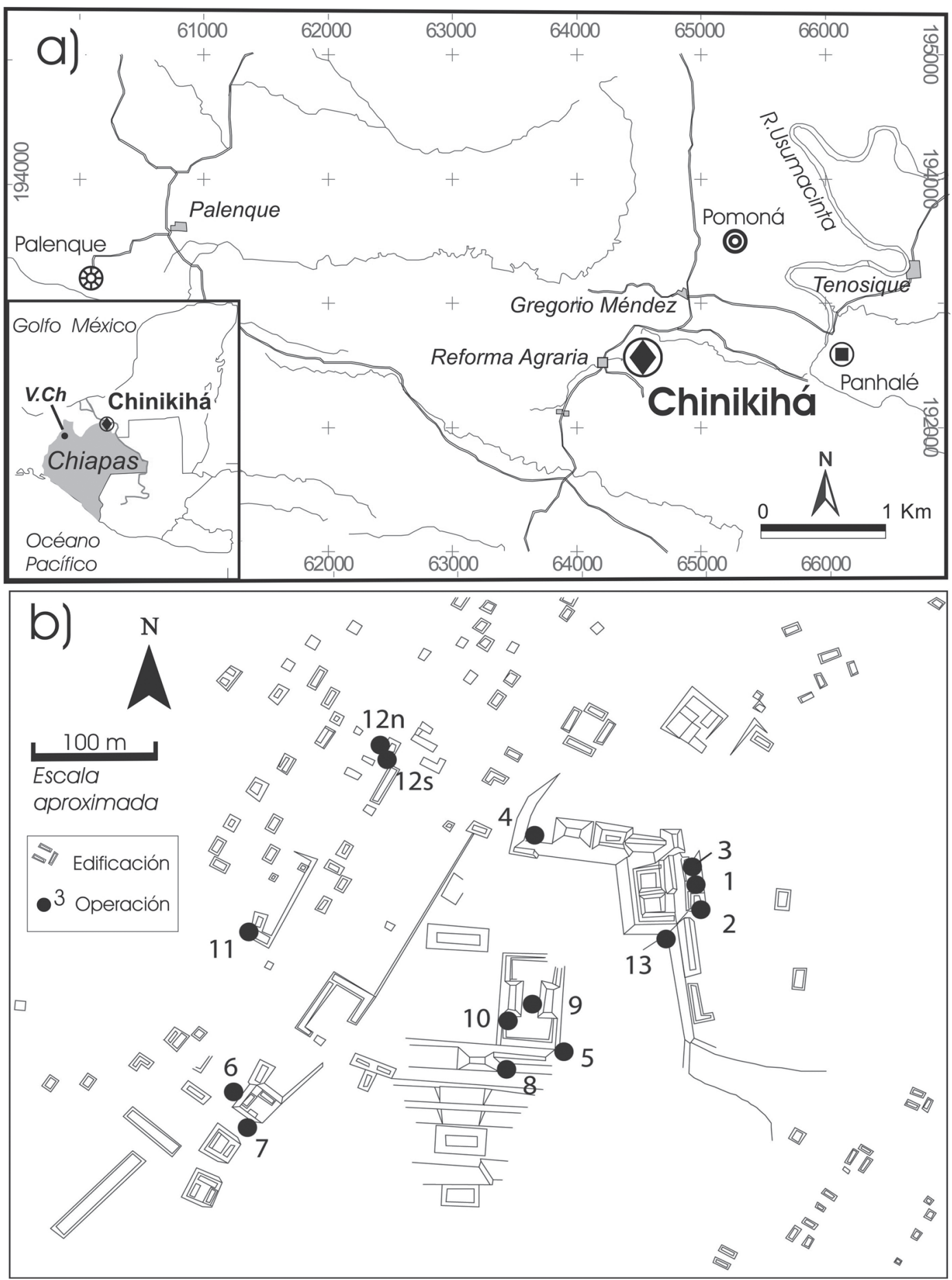

Fig. 1: Mapas de ubicación. a) ubicación del Estado de Chiapas y sitios arqueológicos Chinikihá, Palenque, Chinikihá, Panhalé y Pomoná, poblados y el volcán Chichón (V.Ch). b) esquema de detalle de la zona nuclear del sitio Chinikihá. Los números representan las operaciones arqueológicas, un total de 13 operaciones. 
conformando no menos de 15 grupos orientados a plazas, patios formales e informales (Fig. 1b). Hacia el este, se puede observar una estructura que presenta dos o tres largos pasillos paralelos en dirección norte-sur, los cuales se comunican entre sí mediante diversas cámaras transversales abovedadas. En una de ellas, todavía se conservan restos de pintura mural que se han reportado diversos exploradores y arqueólogos en sus visitas al sitio. También se han registrado un juego de pelota de cancha cerrada que tiene forma de "I", así como la presencia de dos textos, que según Stuart \& Morales (2003), fueron registrados por Maler y confirmados por Greene, Rands, y Graham, quienes mencionan a dos posibles gobernantes locales: K'inich B'ah Tok' y Aj Tok' Ti'. Estos textos mencionan de manera epigráfica a ambos gobernantes como probables líderes de una unidad política independiente en la cuenca media del río Usumacinta (Stuart \& Morales, 2003)

La ubicación misma de Chinikihá es un elemento importante a tomar en consideración, pues se ubica en una de las corrientes ribereñas que comunican la extensa planicie del Golfo de México con el río Usumacinta de Tabasco y Chiapas. Desde el punto de vista cronológico Chinikihá es un asentamiento que conlleva a una problemática que se relacionada con los procesos de formación de las entidades políticas y economías regionales mayas como el señorío de Palenque y otros sitios importantes como Pomoná y Piedras Negras, sin omitir otros asentamientos de la región que aún están siendo investigados (Liendo 2007; López 1991, 2005). Esta comunidad prehispánica fue ocupada de manera importante durante los años 250 al 900 después de Cristo (Jiménez 2009).

De acuerdo a Rands (1967) y Jiménez (2007), las excavaciones y los análisis macroscópicos modales en la zona nuclear, apuntan que Chinikihá es un asentamiento, que durante la primera parte de su historia (mediados del siglo IV), parece haber sido un sitio consumidor, aún no se sabe si productor, de cerámicas de texturas burdas que fueron elaboradas con arcillas, cuyos enseres se caracterizan por la presencia abundante de minerales carbonatados (Rands \& Rands 1957; Rands 1967, 1974, 1987 y 2007; Muñoz 2006; Jiménez
2007, 2009). En la segunda parte de su historia (siglo VIII) y en asociación con los recipientes carbonatados, la cerámica hecha con arcillas de diferentes texturas que contienen una cantidad indiscriminada de cuarzo, son los que predominaron hasta el final de la época de ocupación del asentamiento, tradición cerámica posterior que de acuerdo a la comparación con otros sitios aledaños se relacionaría más con la región de Palenque (San Román, 2005) (Fig. 2). Además es importante mencionar la presencia tardía de formas cerámicas que fueron hechos con arcillas que se constituyen de abundante mica y/o vidrio volcánico. Singularmente en un poco más del $2 \%$ de las unidades arqueológicas excavadas hasta la actualidad, se evidencia que en las materias primas que fueron seleccionadas para la manufactura de tales artefactos cerámicos, existe el reemplazo de pastas carbonatadas por silicoclásticas lo cual ocurrió de manera gradual (Jiménez 2007, 2009).

Entonces una de las preguntas cruciales para comenzar este trabajo fue el de determinar, geoarqueológicamente durante el Clásico de Chinikihá (250-600 d.C.), cuáles son las diferencias petrográficas involucrando la composición, textura, matriz y partículas minerales de las pastas cerámicas.

\section{ASPECTOS GEOLÓGICOS}

Observando las formaciones geológicas en los alrededores del sitio arqueológico, no sorprende la presencia de minerales como cuarzo, plagioclasa, micas y calcita que se presentan de manera habitual en las pastas. Sin embargo, hay que esclarecer que la presencia de vidrios de origen volcánico y la abundancia de micas y de otros minerales no reportados en ciertas formas cerámicas del Clásico Tardío, necesita una determinación de la fuente geológica, así como una explicación de los hábitos culturales en el aprovechamiento de las materias primas arcillosas, sus constituyentes o en el intercambio de recipientes ya elaborados. Aún con el avance en las investigaciones, no es posible discernir si estos patrones culturales estuvieron relacionados con los aspectos políticos y/o económicos de Chinikihá. 

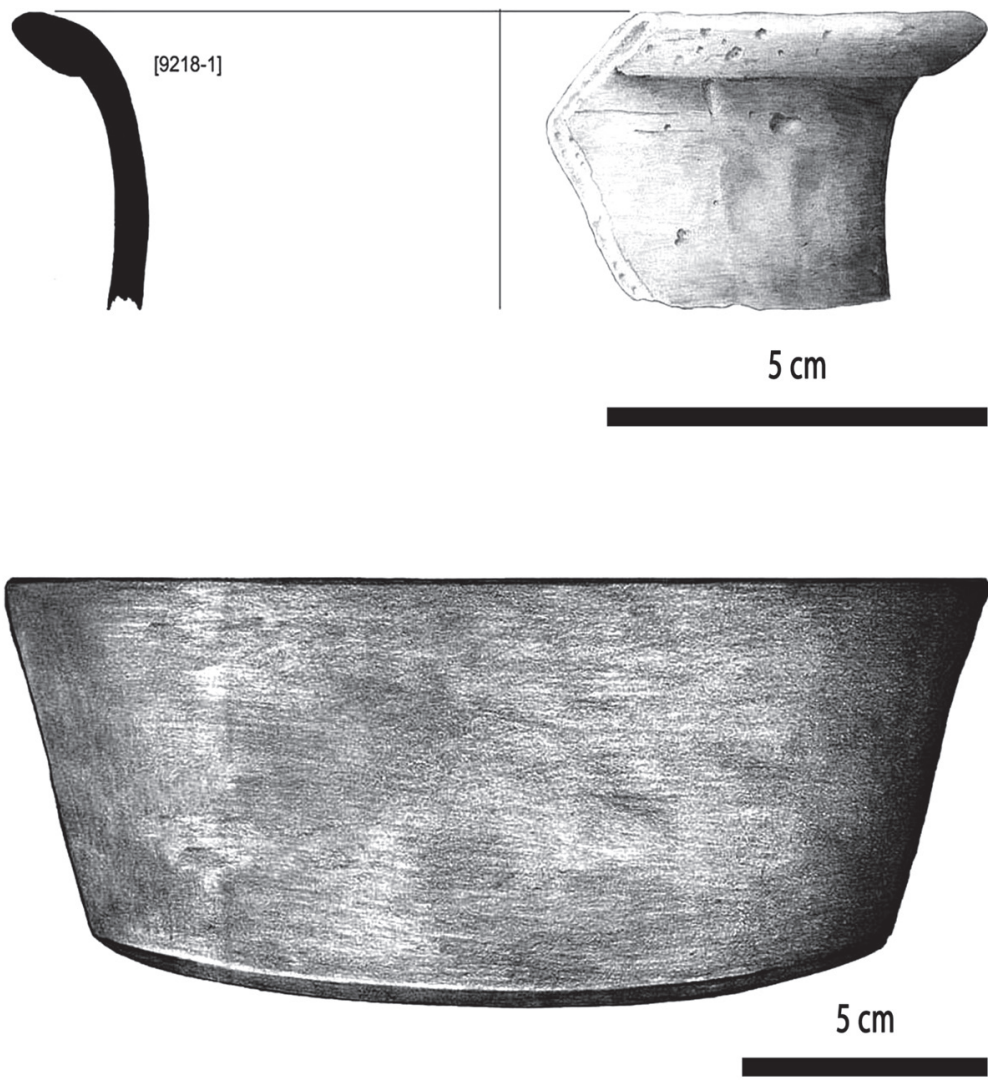

Fig. 2: Formas cerámicas de Chinikihá, según Jiménez, 2009.

\section{Geología}

Geológicamente hablando, Chinikihá esta edificada sobre calizas del Paleoceno (TPal-cz) (Fig. 3), compuesto por mudstone, packstone y grainstone oolíticas, parcialmente recristalizadas y dolomitizadas, color gris oscuro. Afloran en estratos delgados a gruesos y masivos (DGG, 1983). El ambiente fue de plataforma de aguas someras lagunares poco limpias. Su fauna fósil es escasa y mal conservada, representada generalmente por gastrópodos y pelecípodos, aunque hay foraminíferos. Esta secuencia hacia la parte superior puede estar intercalada con lutitas amarillas (TPal-cz-lu). Esta unidad forma las laderas de las sierras con núcleo cretácico (DGG, 1983).
Ligeramente hacia el sur y este de Chinikihá, se encuentran calizas del Cretácico (Ks-cz) (Fig. 3), las cuales son principalmente mudstones con estratos medios a gruesos, de color gris claro a crema, con texturas esparitas, ooesparirudíticas, biomicritas, dismicritas y packstones (DGG, 1983), parcialmente dolimitizadas. Los fósiles indican facies de plataforma con influencia pelágica (INEGI, 1983). Estructuralmente forman los núcleos de los plegamientos anticlinales y fuerte disolución kárstica (INEGI, 1983).

Hacia el sur de Chinikihá, afloran areniscas y lutitas del Eoceno (TE-lu-ar) (Fig. 3), con intercalaciones de areniscas de grano medio, limolitas y lutitas. Las rocas son de color café, rojo y morado (DGG, 1983). Las areniscas son volcaniclásticas de granos angulosos y subredondeados, con fel- 


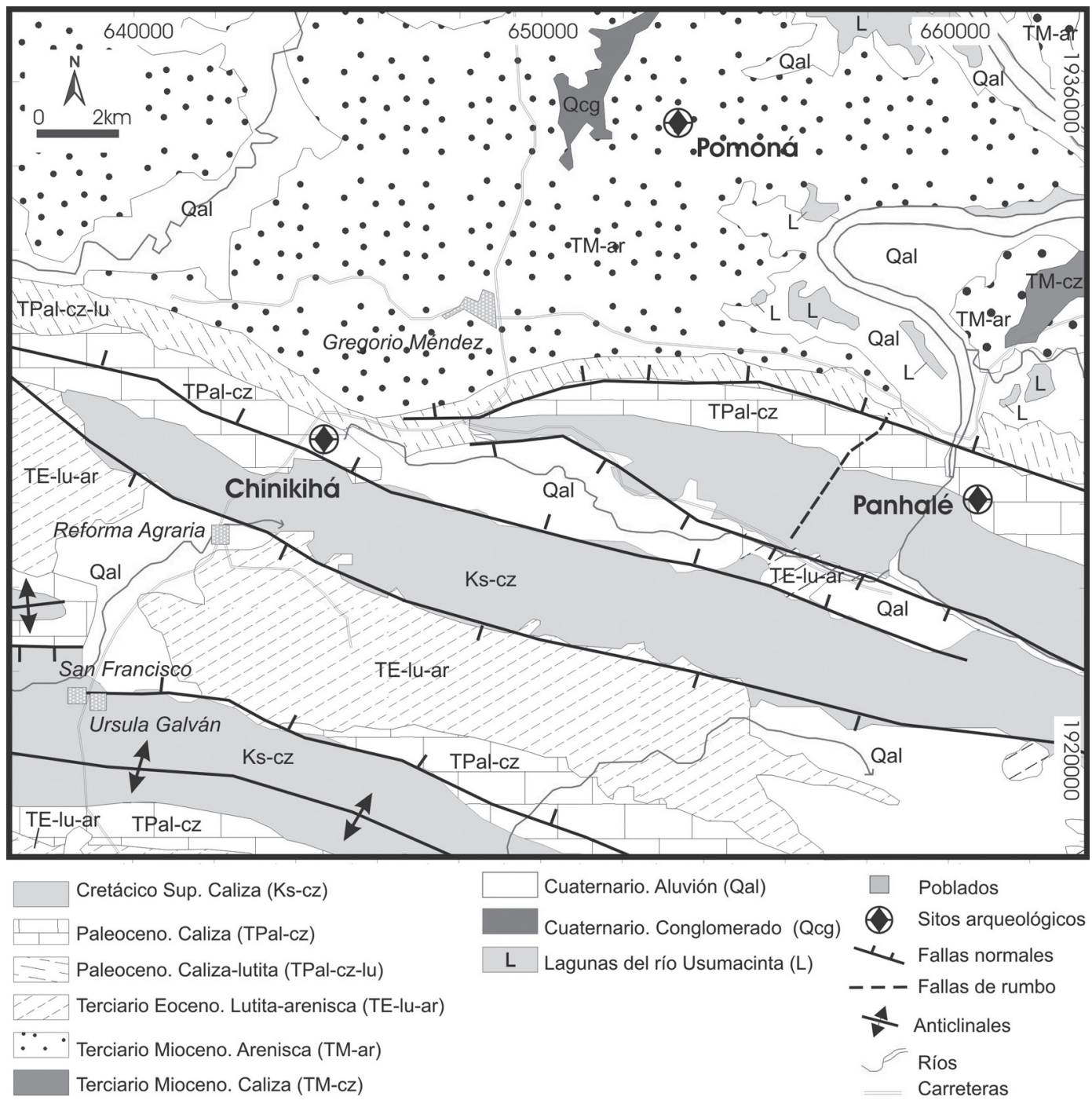

Fig. 3: Mapa geológico, se muestra la ubicación de Chinikihá. Modificado de DGG (1983).

despatos, micas y cuarzos con extinción ondulante. Las limolitas son calcáreas, con un calor variable de café rojizo, a gris verdoso, finamente estratificadas. La depositación fue continental y de ambiente de transición (DGG, 1983).

Cabe destacar que hacia las planicies meándricas del Usumacinta, al norte de Chinikihá, se encuentran extensas zonas planas y pantanos formados sobre areniscas del Mioceno (TM-ar) (Fig. 3), las cuales están poco consolidadas, meteorizadas (INEGI, 1983) y compuestas por litoarenitas de grano medio que contienen cuarzo, muscovita, feldespatos, circón, caolín y fragmentos de roca en una matriz arcillosa, medianamente cementadas por hematita y sílice. Se presentan en estratos muy deleznables de 1 a $5 \mathrm{~m}$ de espesor o masivos, color amarillo, café oscuro y rojo. Se puede observar intercalaciones de lutitas, limolitas y calizas. El paleoambiente del depósito fue transicional de intramarea (DGG, 1983; INEGI, 1983).

La geología estructural está marcada por un fuerte plegamiento de orientación casi este-oeste, con anticlinales y sinclinales (Fig. 3), isopacos, 
asimétricos, en forma de cofre o invertidos. El plegamiento se originó desde el Cretácico tardío hasta el Eoceno, correspondiente con la orogenia Laramídica (INEGI, 1983).

El fallamiento también tiene una dirección este-oeste paralelo al plegamiento. En el área de Chinikihá, el fallamiento es normal, dejando estructuras tipo graben y pilares. El fallamiento transcurrente es el último evento tectónico que data del Plioceno hasta el Reciente (INEGI, 1983).

\section{ANÁLISIS PETROGRÁFICO}

La petrografía permite ahondar en el conocimiento de los contenidos mineralógicos, texturas, fábricas y los procesos de manufactura, tratando de determinar modos diagnósticos útiles en la clasificación e identificación de cerámicas. Cabe mencionar que durante esta etapa de identificación de componentes, no se hacen inferencias respecto a lo que podrían ser los agregados naturales o intencionales (lo que en términos arqueológicos algunos llaman inclusiones naturales vs. desgrasante o refractario). Este último punto aún es un tema de discusión en lo que se refiere a los estudios de tecnología cerámica preshipánica.

Se analizaron petrográficamente 21 muestras de fragmentos de cerámicas obtenidos de las 13 operaciones estratigráficas que fueron excavadas durante la temporada de campo de 2005 (Fig. 1b).

\section{Contenido mineralógico}

Se identificaron un total de 16 minerales, entre estos se pueden indicar los grupos de óxidos, carbonatos, sílices, fosfatos, feldespatos, anfíboles, micas, cloritas, amorfos como los vidrios de formas variadas y fragmentos de roca (Cuadros 1 y 2). Se puede indicar que los minerales más abundantes y comunes son cuarzos (por lo general tienen extinción ondulante y en algunas ocasiones son policristalinos), calcita y hematita (Fig. 4a y 4b); como componente fuera de lo común aparecen fósiles de diatomeas.
Desde el punto de vista de la ausencia, presencia, abundancia y asociación relativa de los minerales identificados en las secciones delgadas, se pueden diferenciar perfiles petrográficos o grupos de referencia. En las muestras analizadas se pueden clasificar en dos grupos principales (Cuadro 1).

Aunque su porcentaje sea variado, todas las muestras presentan una matriz arcillosa soportando los granos, lo cual es obvio debido a la naturaleza cerámica. Sin embargo, en lo que respecta a los agregados, se observaron que las cerámicas pueden tener dos tendencias, a) las cerámicas en las cuales dominan los carbonatos y presentan minerales subordinados como cuarzo, feldespatos, fragmentos de roca, etc, y b) aquellos en que el componente silicoclástico es el componente dominante (aluminosilicatos) y el carbonato es muy subordinado o está ausente.

A continuación se describen los dos criterios principales de clasificación general petrográfica:

1) Clasificación general petrográfica, basada en el contenido de matriz y minerales principales.

2) Clasificación con parámetros de la textura $\mathrm{y}$ formas de los granos.

\section{Clasificación General Petrográfica}

El Cuadro 1 muestra dos tendencias de componentes dominantes: primero, los componentes silicoclásticos (con 45,5 al 84,5\% de matriz con un promedio de 69,19\%) en donde los componentes dominante son los aluminosilicatos y la segunda tendencia es el componente carbonatado con un contenido de matriz entre el 18 al $64 \%$, cuyo promedio es de $30,5 \%$ y carbonato de hasta $79 \%$.

\section{Matriz}

El porcentaje de matriz, su distribución y fábrica, es un modo diagnóstico clasificatorio utilizado en esta investigación. El término matriz se refiere a la arcilla como medio soportante de los granos, expresado en porcentaje por volumen de muestra. Debido a la temperatura de cocimiento de la cerámica, la arcilla, así como otros minerales 
a) Silicoclástico, porcentaje de matriz y agregados

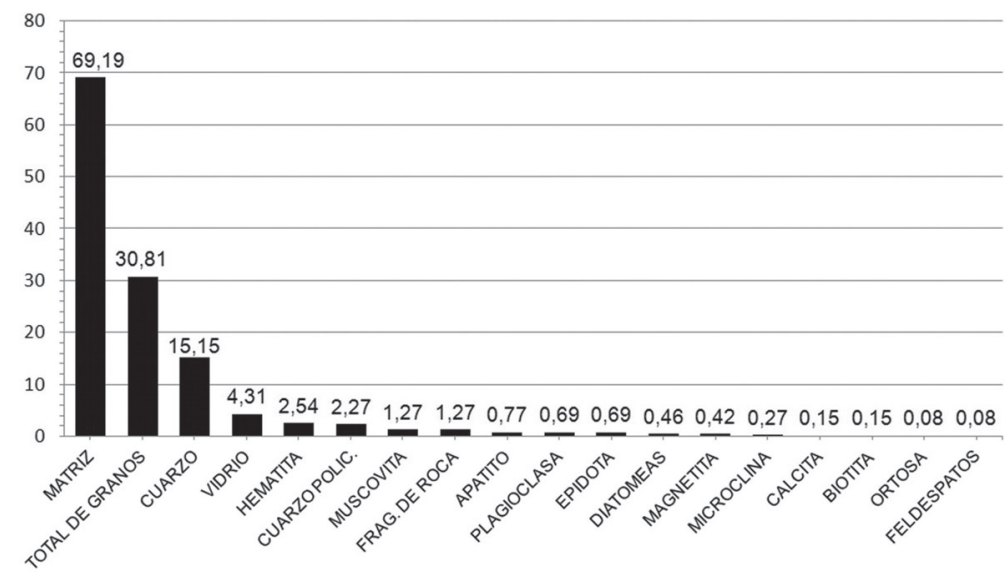

b) Carbonatada, porcentajes de matriz y agregados

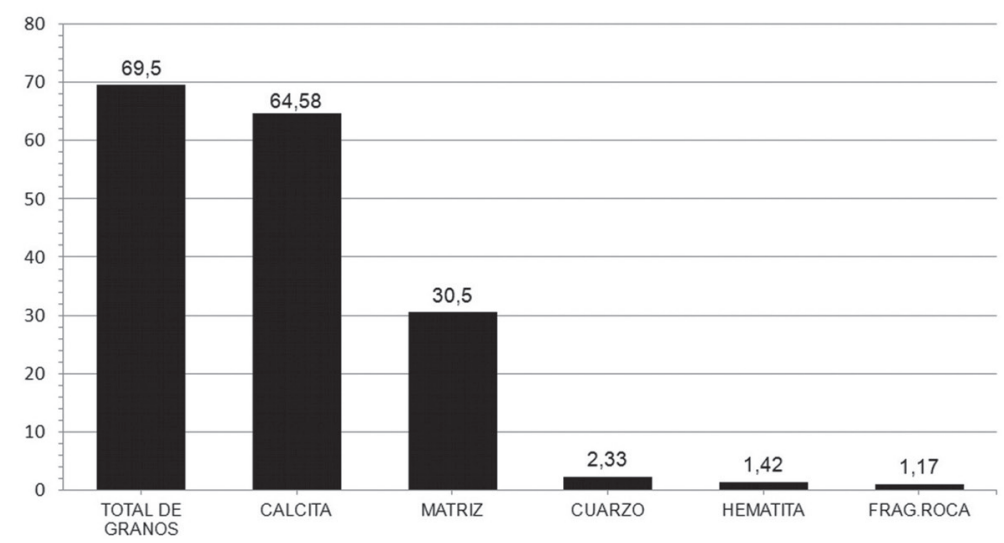

Fig. 4: Gráficos mostrado porcentajes de granos y matriz, basado en el análisis petrográfico. a) cerámica Silicoclástica, siendo el cuarzo y el vidrio volcánico los más abundantes. b) cerámica Carbonatada, siendo los granos de calcita los clastos dominantes.

experimentarán transformaciones o recristalizaciones por altas temperaturas (Deer et al.,1966, Ervan \& Garrison, 1998).

La composición mineral de la matriz no fue determinada, pero petrográficamente es posible observar el grado de filomorfismo, esto es, el contenido de minerales filosilicatados. Esto hace que la matriz adquiera birrefringencia bajo luz polarizada con un grado de filomorfismo de bajo a alto. Los valores del contenido arcilloso en la matriz varían entre $18 \%$ al $84,5 \%$.

\section{Clasificación basada en parámetros de textura y formas de los granos}

Los parámetros petrográficos adicionales utilizados en la descripción y elementos del modo diagnóstico, son los siguientes: minerales presentes, aspectos granulométricos además de redondez, esfericidad, selección, tipo de contactos. 
Cuadro 1

Se muestra las dos tendencias de grupos, los componentes silicoclásticos y carbonatados, así como otros componentes, todos englobados en matriz arcillosa

\begin{tabular}{|c|c|c|}
\hline \multirow{3}{*}{ Matriz arcillosa $18-84,5 \%$} & Grupos & Otros componentes \\
\hline & $\begin{array}{l}\text { Grupo 1: Componentes silicoclás- } \\
\text { ticos } \\
45,5-84,5 \% \text { matriz, promedio } \\
69,19 \%\end{array}$ & $\begin{array}{l}\text { Cuarzo, fragmentos de roca, feldes- } \\
\text { patos, micas, vidrio, calcita, epi- } \\
\text { dota, etc. }\end{array}$ \\
\hline & $\begin{array}{l}\text { Grupo } 2 \text { : componentes carbonata- } \\
\text { dos } \\
18,64 \% \text { matriz, promedio } 30,5 \%\end{array}$ & $\begin{array}{l}\text { Cuarzo, fragmentos de roca, óxi- } \\
\text { dos, etc. }\end{array}$ \\
\hline
\end{tabular}

\section{Grupo 1: Silicocláticos}

Este grupo representado en el Cuadro 2 y Fig. $5 \mathrm{a}$, se caracteriza porque no contiene carbonatos dominantes y el mayor componente es el cuarzo; el resto son minerales subordinados.

Particularmente la muestra UY0221 (un cuenco), macroscópicamente fue clasificada dentro del grupo de pastas arenosas, pero se diferencia del resto de estas pastas ya que al tacto da una sensación gredosa, además por su color anaranjadorojizo y por tiznar los dedos al frotar la superficie. De igual manera respecto a la dureza, resulta ser mucho menos resistente que el resto de las pastas arenosas. Las características físicas que se indicaron anteriormente se deben a una mayor proporción de óxidos identificados durante el análisis microscópico. Tiene abundancia de cuarzo y calcedonia. En este ejemplar se incrementa la cantidad de óxidos (hematita y magnetita).

En el caso de la muestra UY0212 (un cajete). Esta se distingue del resto de los ejemplares debido a la abundancia de cuarzo, calcedonias y epidotas y los componentes subordinados son: fragmentos de roca, microclinas, muscovitas y ortosa, los cuales son componentes únicos para esta muestra.

Granulométricamente hablando, los agregados de las cerámicas silicoclásticas varían desde limos finos a arenas medias. Respecto a la redondez de los agregados, por lo general las muestras presentan granos angulosos con baja esfericidad aunque localmente son subredondeados de alta esfericidad.

Las muestras UY0220, UY0227, UY0240, UY0238 se caracterizan únicamente por la presencia de los cuarzos y otros minerales subordinados con poca abundancia como en el caso de las microclinas, calcedonias, epidotas, muscovitas, óxidos y fragmentos de rocas meteorizados.

Como observaciones mineralógicas, en este grupo, los cuarzos tienen extinción ondulante y pueden aparecer cuarzos policristalinos de probable origen metamófico o sedimentarios. Por otra parte, en otras secciones las plagioclasas (UY0238) muestran sericitización.

Se considera que la presencia de cuarzo por sí solo no tiene relevancia significativa en las pastas de Chinikihá, debido a que los cuarzos son minerales detríticos en las arcillas (materia prima) que fueron empleadas por los alfareros de este asentamiento. El cuarzo podría ser un elemento que defina un modo diagnóstico cuando éste alcance porcentajes significativos en la cerámica. Pero por el momento, debe ser considerado tentativamente, como un mineral de poca importancia en la separación de los perfiles petrográficos. Por otra parte, dado que el subgrupo cerámico de los silicoclásticos con apatitos (Grupo 1c) tiene una composición petrográfica similar al grupo de cuarzos, se hace necesario un estudio más detallado de este último mineral. 
En la muestra UY0227, se identificaron fragmentos de roca volcánica muy meteorizados, así como microclinas que se muestran arcillitizadas (meterorizada). Esta sección delgada corresponde a un fragmento de cuenco que tiene una textura rasposa al tacto, debido a que las partículas quedaron expuestas al erosionarse la superficie del tiesto, probablemente cuarzo $(10 \%)$.

\section{Grupo 1a: Grupo de los silicoclásticos con vidrio}

Grupo de muestras que presentan entre sus componentes partículas abundantes de vidrios volcánicos (hasta un 25\%, Cuadro 2) (Fig. 5b, Fig. 6a y 6b) y de cuarzos policristalinos de probable origen metamórfico y sedimentario. Aunque las microclinas están presentes en este grupo, su presencia es poco abundante (menos del 1\%). También se puede mencionar la presencia de otros componentes, como por ejemplo los fragmentos de roca epidota, muscovita (Fig. 6c). Las magnetitas y las plagioclasas se identificaron específicamente en la muestra UY0233. Se puede indicar que la muestra UY0247 se diferencia poco significativamente del resto de las muestras, por la presencia de los fragmentos de roca contenidos en la pasta ( $1,5 \%$ máximo).

Como información mineralógica adicional se puede mencionar que los cuarzos policristalinos de origen metamórfico caracterizan a algunas de las secciones delgadas (UY0233, UY0241). Como caso particular, en la muestra UY0241 se pudo observar muscovita asociada al cuarzo como parte de un fragmento de roca metamórfica de textura poikiloblástica. Los cuarzos presentan extinción ondulante. El contenido de vidrio (hasta un $25 \%$, ) tiene formas de yunque, trípodes, cuñas, agujas, en forma de oz y glóbulos completos o semicompletos así como otras formas variadas (Fig. 6a y 6b).

Desde el punto de vista general, los granos se caracterizan ser muy angulosos con baja esfericidad y angulosos con alta esfericidad, subangulosos con baja esfericidad. No se observa ningún patrón homogéneo. El tamaño del grano mayormente es limo medio hasta arena fina.
La selección de los granos tiende a ser buena. Respecto al empaquetamiento, dominan los contactos flotantes y subordinados puntuales.

La cerámica clasificada macroscópicamente como Arenosa bayo, Arenosa y Caolinita, petrográficamente, se ubican dentro del Grupo Silicoclástico con Vidrio. La Arenosa bayo y la Caolinitica (Cuadro 3), son cerámicas que visualmente muestran una textura extremadamente fina. A simple vista no se les observa partículas en la pasta (Jiménez, 2009).

\section{Grupo 1b: Grupo de los silicoclásticos con micas}

Las pastas de este grupo clasificadas como micáceas muestran una brillantez debido de los espéculos contenidos en la superficie y en la pasta. Únicamente las ollas de cuello bajo que no han sido fechadas de manera definida en los contextos excavados en la zona nuclear de Chinikihá caracterizan a la muestra de este grupo de referencia. El aspecto general de la petrografía se muestra en la figura $5 \mathrm{c}$.

El Grupo 1b está representado por la muestra UY0265 (Cuadro 3), esta olla, representan el 80\% de la cazuelas de Chinikihá. Entre sus componentes se observan abundantes partículas de cuarzo con extinción ondulante (23\%) y muscovitas $(7 \%)$. En menor proporción, se presentan las plagioclasas y la calcita, en tanto que las microclinas, plagioclasas y la calcedonia son subordinados. Los feldespatos muestran una marcada sericitización.

La redondez de los granos está entre muy anguloso de baja esfericidad y redondeado de alta esfericidad; el tamaño del grano es una arena fina a media bien seleccionada. Respecto al empaquetamiento, abundan los contactos puntuales y los subordinados son los flotantes largos.

\section{Grupo 1c: Silicoclástico con apatito y diatomeas}

Las muestras UY0208 (cajete) y UY0211 (cuenco), se caracterizan por presentar una abundancia de fragmentos de cuarzo, apatito y diatomeas (Cuadro 2 y Fig. 5d). En cantidades menos abundantes le seguirían la muscovita y la calcedonia. Fragmentos de roca, plagioclasas, 

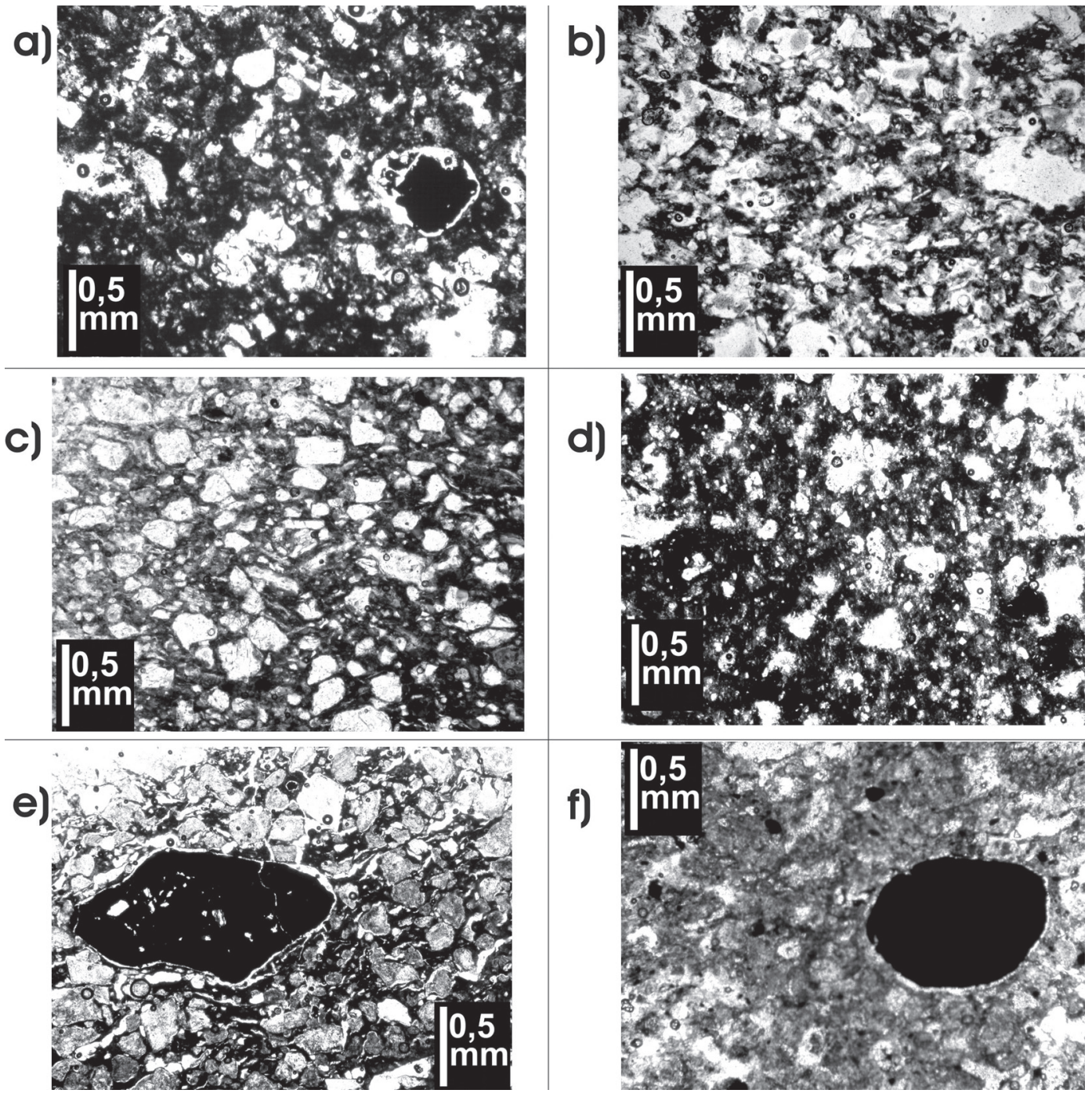

Fig. 5: fotografías de secciones delgadas, cerámicas Chinikihá. Nícoles paralelos. a) grupo 1, silicoclástico. b) grupo 1a, silicoclástico con vidrio. c) grupo $1 \mathrm{~b}$, silicoclástico con micas. d) grupo $1 \mathrm{c}$, silicoclástico con apatito y diatomeas. e-f) grupo 2, calcita, con fragmentos de roca (en negro).

microclina, hornblenda y epidota se pueden considerar como componentes poco significativos. Como observaciones mineralógicas se menciona que en ambas secciones delgadas, los cuarzos son policristalinos de origen metamórfico o sedimentario, en tanto que los fragmentos de roca se observaron hematitizados. Es importante señalar que los cuarzos también mostraron una extinción ondulante. Un rasgo muy significativo en estas cerámicas, es la pre- sencia de fósiles de diatomeas cilíndricas o con frústulas tipo Pennales, las diatomeas tienen un caparazón de sílice amorfo (ópalo). Esto hace pensar que la fuente de la pasta cerámica fue cercana a lagos o lagunas de agua dulce (Fig. 7a, $7 \mathrm{~b}$ y 7c). Observando los mapas geológicos (Fig. 3) y fisiográficos de Chiapas, es notoria la presencia de muchas áreas pantanosas colindantes al río Usumacinta; estos humedales empiezan a aparecer a los $15 \mathrm{~km}$ hacia el NW 


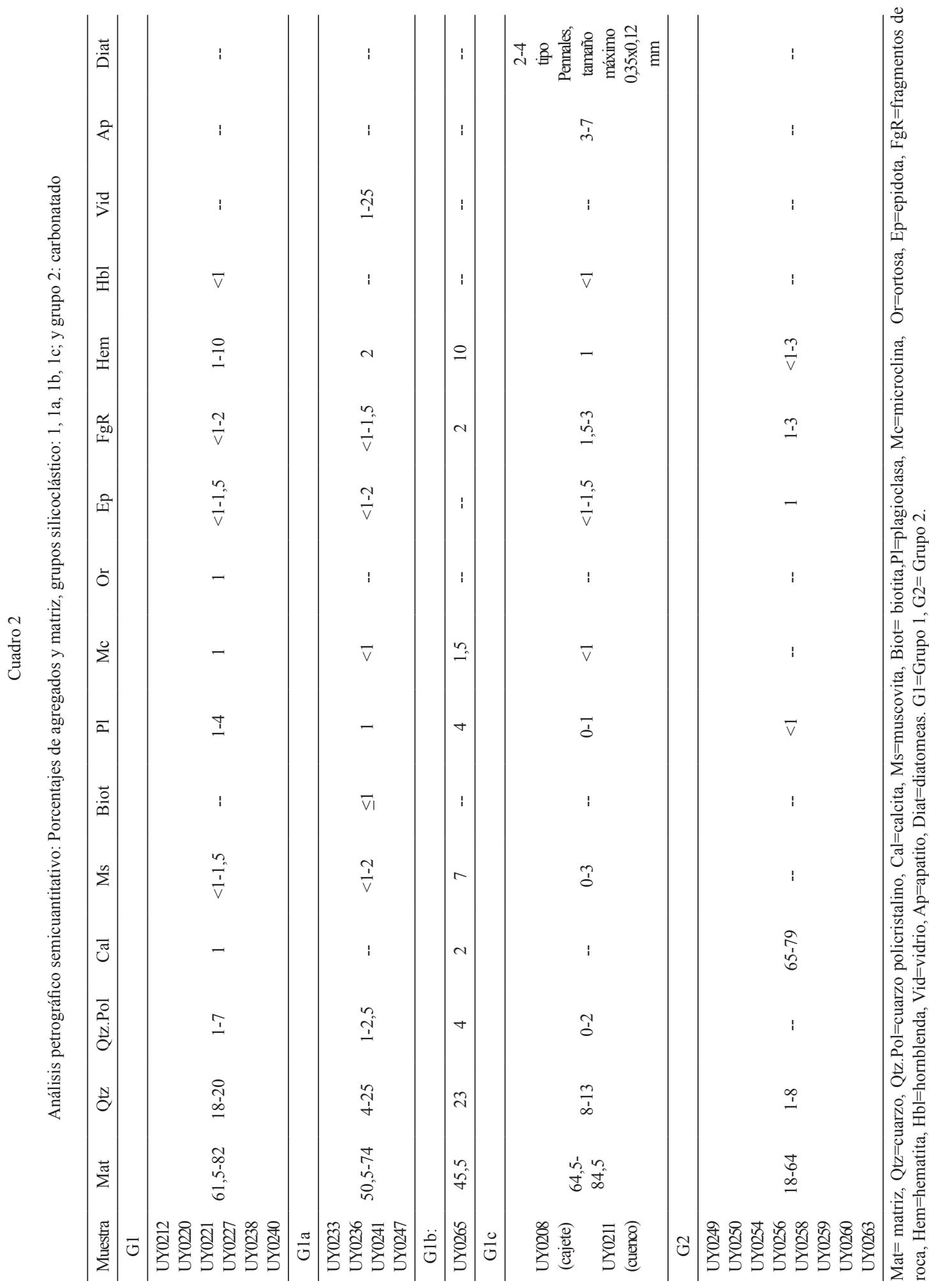


de Chinikihá, quizás la materia prima de estas pastas provenían de dichos humedales.

Desde el punto de vista general, los granos angulosos son de baja y alta esfericidad hasta subredondeados con alta esfericidad, no se observa ningún patrón homogéneo. El tamaño del grano mayormente es limo, sin embargo, se observaron granos desde arena fina hasta arena gruesa. La selección de los granos tiende a ser mala a muy mala. El empaquetamiento dominan los contactos flotantes y subordinados puntuales.

\section{Grupo 2: Calcita con matriz arcillosa}

El cuadro 2, muestra los componentes principales de este grupo, presenta fragmentos de calcita y hematites con un soporte de arcilloso (Fig. 5e y 5f). Nótese la abundancia de calcita $(65-79 \%)$ y el rango de matriz arcillosa ( $18 \%$ a $64 \%$ ), con un promedio de $30,5 \%$, por lo general el filomorfismo de la arcilla es de bajo a medio. Se destacan las muestras UY0263 y UY0249, esta última contiene un $64 \%$ de arcilla pero el componente dominante detrítico es la calcita con $35 \%$ y tan solo $1 \%$ de fragmentos de roca. La muestra UY0263 alcanza el valor más bajo de matriz (18\%), la calcita detrítica es de $71 \%$ con un $8 \%$ de cuarzo, aquí prácticamente el soporte es por grano.

Los cuarzos, de extinción ondulante, son componentes principales que se presentan en menor proporción que las calcitas En la muestra UY0249 se observan algunos fragmentos de plagioclasas.

Las muestras UY0254 y UY0256 se podrían considerar como referencias diagnósticas debido a la ausencia de cuarzos o de otros minerales que no sean los óxidos. Sin embargo, para poder corroborar dicha aseveración, se considera preciso ampliar el rango de análisis de las muestras petrográficas en este grupo de calcitas. Ya que el ejemplar UY0256 procede de contextos que han sido fechados por su asociación relativa con otros materiales diagnósticos de la Fase Otolum, o sea el periodo de ocupación prehispánica que se relaciona con el Complejo cerámico Otolum de Palenque, el cual ha sido fechado entre el 600 y el 700 d.C. Chinikihá tiene relaciones cerámicas estilísticas y modales con esta fase.
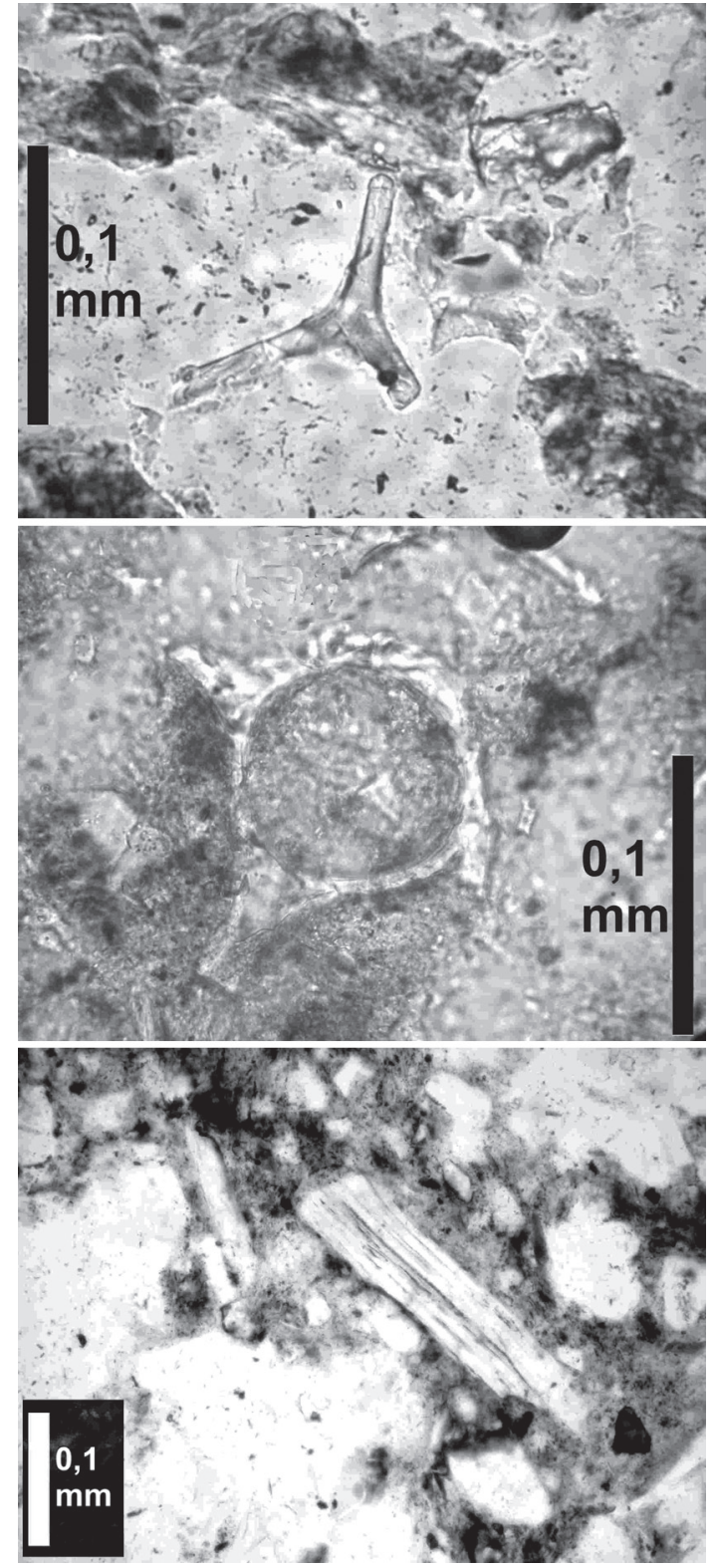

Fig. 6: a) muestra UY 0233. Esquirla de vidrio en forma tripoide. b) Muestra UY 0241. Esquirla de vidrio globular. c) Cristal de muscovita, rodeado por cuarzo, ligeramente deformado por compactación de la pasta.

Desde el punto de vista general, la redondez y esfericidad se caracteriza por ser angulosos de baja esfericidad y en menos proporción angulosos con alta esfericidad, resultando muy homogénea 
(Fig. 5e y 5f). El tamaño del grano varía desde limo grueso hasta arena media. La selección de los granos es de moderada a bien seleccionada. Dominan los contactos puntuales y largos con contactos flotantes subordinados.

\section{CONSIDERACIONES}

a) Sobre el Filomorfismo: la presencia de filosilicatos en las muestras analizadas puede representar una recristalización de las arcillas utilizadas como materia prima. Debido a su tamaño, estos filosilicatos observados en las muestras de Chinikihá, no pueden ser identificados petrográficamente, ya que se requerirá de otros análisis para su identificación, por ejemplo, vía rayos X. Deberá comprobarse si estos filosilicatos son illitas o micas, dado que las illitas tienen una estructura cristalina similar a las micas. Estas illitas pueden ser estables aún a $950^{\circ} \mathrm{C}$, más allá de este rango desaparecen (Ervan \& Garrison, 1998).

b) Muestras con Feldespatos-K: Los feldespatos de potasio (ortosas, microclinas), son estables hasta los $950^{\circ} \mathrm{C}$ (Ervan \& Garrison, 1998), después de este rango, estos minerales desaparecen.

c) Muestras con Carbonato dominante: De acuerdo a Ervan \& Garrison (1998), las muestras carbonatadas probablemente, no fueron calentadas a mas de $800^{\circ} \mathrm{C}$, esto debido a que más temperatura el carbonato se oxida y desaparece o se convierten en piroxenos.

d) Esquirlas de vidrio volcánico: Los beneficios tecnológicos que se obtenían del uso del vidrio en las cerámicas fueron ventajosos con respecto al uso de la calcita. Se dice que los fragmentos de vidrio son aditivos estables y la superioridad de las cenizas volcánicas con vidrio respecto a otros agregados determinaron su extensivo uso (Shepard 1976). Por otra parte, la forma angular de las partículas confiere estabilidad a la arcilla durante el formado de las piezas (Shepard 1964, Shepard 1976).

La presencia del vidrio volcánico (shards) en las cerámicas, es un tema de investigación antropológica, de acuerdo a Simmons \& Brem (1979),

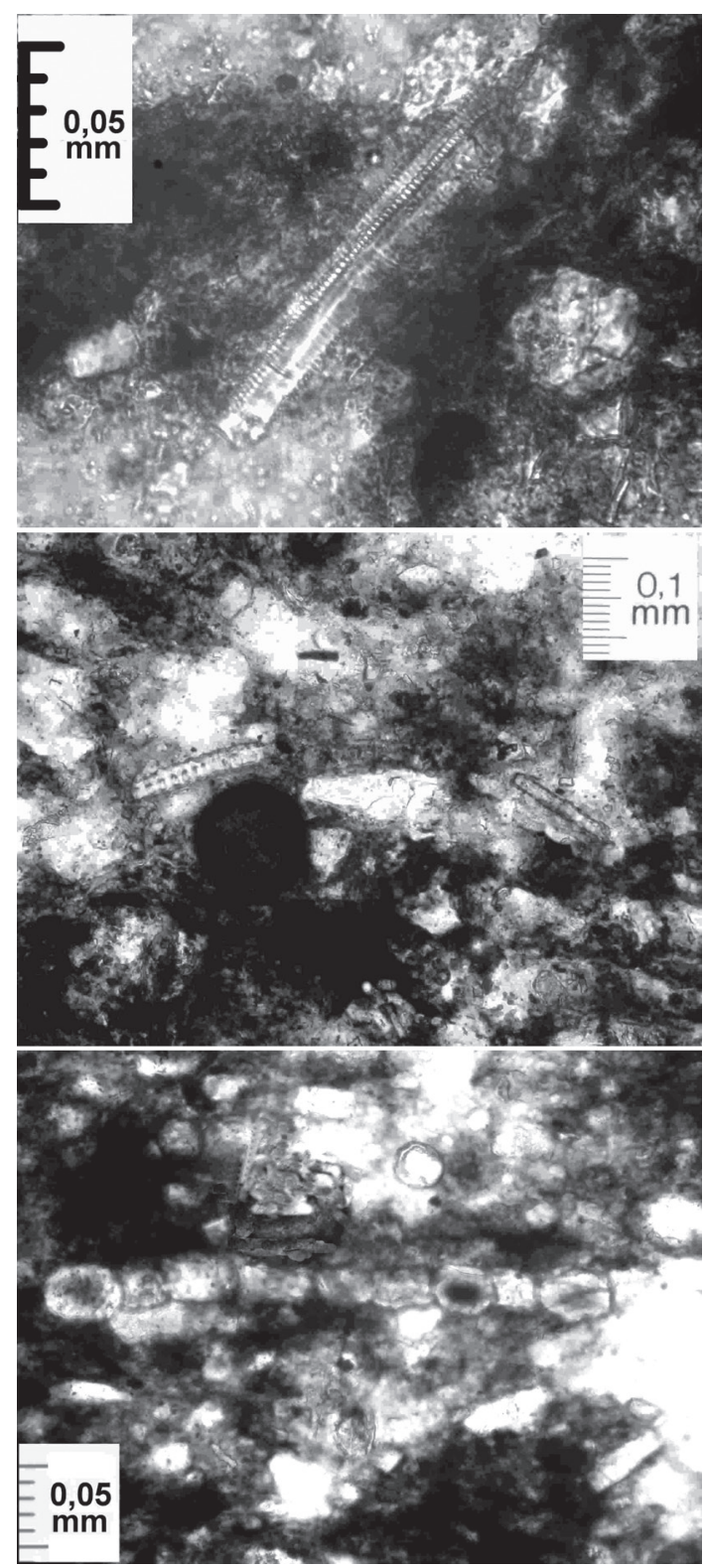

Fig. 7: a) fósil de diatomea, en donde se observan las frústulas dentadas. Muestra UY0208. b) fósil de diatomea, con las frústulas dentadas. Muestra UY0208. c) fósil de diatomea, cadenas cilíndricas. Muestra UY0208.

Ford \& Rose (1995), Ford \& Spera (2007) y Chung, (2009), no se tiene claro cuál es el origen de la materia prima silicoclástica que contiene las esquirlas de vidrio. Ford \& Rose (1995), sugieren que quizás regularmente los volcanes Chichón 
Cuadro 3

Clasificación macroscópica de las pastas cerámicas: Grupo petrográfico y Clasificación macroscópica,Chinikihá.

\begin{tabular}{|c|c|c|c|c|}
\hline Código & Grupo petrográfico & $\begin{array}{l}\text { Clasificación } \\
\text { macroscópica }\end{array}$ & Forma & Descripción \\
\hline UY 0220 & Grupo 1 & Arenosa & cuenco & $\begin{array}{l}\text { Pasta de textura mediana que mues- } \\
\text { tra diferentes tonalidades de color } \\
\text { amarillo-marrón, marrón amarillo } \\
\text { claro. Son comunes los núcleos de } \\
\text { color gris oscuro }\end{array}$ \\
\hline UY 0212 & Grupo 1 & Negro fino & cajete $(*)$ & Idem descripción UY 0220 \\
\hline UY 0221 & Grupo 1 & Arenosa & cuenco & Idem descripción UY 0220 \\
\hline UY 0227 & Grupo 1 & Arenosa & cuenco & Idem descripción UY 0220 \\
\hline UY 0238 & Grupo 1 & Pomácea $(+)$ & olla & Pasta de textura burda de color marrón \\
\hline UY 0240 & Grupo 1 & Marrón fina & cajete $(*)$ & Pasta de textura fina de color gris rosado \\
\hline UY 0233 & Grupo 1a & Arenosa & cazuela & Idem descripción UY 0220 \\
\hline UY 0236 & Grupo 1a & Arenosa bayo & plato & Pasta de textura fina de color marrón claro \\
\hline UY 0241 & Grupo 1a & Caolinítica & plato & $\begin{array}{l}\text { Pasta de textura fina de color } \\
\text { marrón-claro }\end{array}$ \\
\hline UY 0247 & Grupo 1a & Arenosa & cazuela & Idem descripción UY 0220. \\
\hline UY 0265 & Grupo $1 \mathrm{~b}$ & Micácea & olla & Pasta de textura mediana color marrón \\
\hline UY 0208 & Grupo 1c & Negro fino & cajete $(*)$ & Pasta de textura fina de color marrón claro \\
\hline UY 0211 & Grupo $1 \mathrm{c}$ & Negro fino & cuenco & Pasta de textura fina de color marrón claro \\
\hline UY 0249 & Grupo 2 & Carbonatada & olla & $\begin{array}{c}\text { Pasta de textura burda de color rosa } \\
\text { a marrón-rojizo }\end{array}$ \\
\hline UY 0250 & Grupo 2 & Carbonatada & sahumador $(* *)$ & Idem descripción UY 0249 \\
\hline UY 0254 & Grupo 2 & Carbonatada & olla & Idem descripción UY 0249 \\
\hline UY 0256 & Grupo 2 & Carbonatada & olla & Idem descripción UY 0249 \\
\hline UY 0258 & Grupo 2 & Carbonatada & olla & Idem descripción UY 0249 \\
\hline UY 0259 & Grupo 2 & Carbonatada & olla & Idem descripción UY 0249 \\
\hline UY 0260 & Grupo 2 & Carbonatada & incensario & Idem descripción UY 0249 \\
\hline UY 0263 & Grupo 2 & Carbonatada & sahumador $(* *)$ & Idem descripción UY 0249 \\
\hline
\end{tabular}

(+)pomácea: pasta de textura burda, $\left(^{*}\right)$ cajete: recipiente de cerámica de boca ancha. $(* *)$ sahumador: recipiente de cerámica de función ritual para sahumar resinas o dar humos aromáticos a fin de purificar

y Cerro Quemado, localizados en las tierras altas mayas, han aportado cenizas de caída; estos volcanes estuvieron activos durante el Periodo Clásico Tardío. De acuerdo a Macías (2005), los flujos piroclásticos del volcán Chichón producidos en la erupción de hace 2400 años (erupción pliniana), contenían fragmentos de cerámica casera, lo que claramente indica que las faldas del volcán habían sido habitadas durante esos últimos 2500 años. También se reportaron fragmentos cerámicos y objetos de obsidiana en las tefras del Chichón datadas entre los 2500 y 2290 años, luego entre los 1250 y 900 años (Macías, 2005). Nooren, et al., (2008), confirman que las sociedades prehispánicas de las zonas bajas Mayas fueron afectadas por repetidas caídas de ceniza de las erupciones del volcán Chichón.

Dado lo anterior y debido a la relativa cercanía de Chinikihá (167 km hacia el NNE), con la zona de influencia del volcán Chichón, es dable suponer que los vidrios encontrados en las cerámicas con pastas silicoclásticas, proceden de los 
productos piroclásticos de dicho volcán, máxime que durante el Clásico Tardío, tal y como lo indican Simmons \& Brem (1979), el comercio de materia prima conteniendo ceniza volcánica, se incremento significativamente.

La confirmación de nuestra hipótesis de trabajo, en donde los vidrios de Chinikiha proceden del volcán Chichón, requiere análisis químicos comparativos entre los vidrios del volcán Chichón y las esquirlas de vidrio contenido en las pastas cerámicas de Chinikihá. Por último, la presencia recurrente de estos materiales en la sierra de Chiapas, son problemas de investigación actual.

\section{CONCLUSIONES}

Los resultados de las secciones delgadas analizadas en Chinikihá basados en los parámetros de la composición y aspectos de la textura indican las siguientes conclusiones:

El repertorio de pasta de Chinikiha muestra una buena variedad de perfiles petrográficos a través de sus distintos periodos de ocupación humana y alcanzan una buena diversidad con las pastas silicoclásaticas.

Se considera que las pastas cerámicas carbonatadas fueron las más tempranas, luego dichas pastas fueron sustituidas gradualmente por las pastas silicoclásticas. El cambio tecnológico y de mentalidad en el uso de cerámicas, probablemente ocurrió al sustituir las pastas carbonatas por pastas silicoclásticas.

Para este análisis, el $80 \%$ de las cazuelas de Chinikiha proceden del grupo silicoclástico con micas dominantes.

El soporte por matriz es más notorio en las pastas silicoclásticas, mientras que las pastas carbonatadas tienden a tener un soporte por grano.

Aunque las temperaturas de cocción exactas no se conocen, se puede inferir que las temperaturas máximas en las cerámicas silicoclásticas probablemente no superaron $\operatorname{los} 950^{\circ} \mathrm{C}$, mientras que las cerámicas carbonatadas no pudieron alcanzar los $800^{\circ} \mathrm{C}$.

Si la materia prima para la manufactura de las cerámicas no fue importada, se infiere que el origen de esta materia prima puede ser las areniscas y lutitas de edad Eocena (TE-lu-ar), o bien las areniscas del Mioceno (TM-ar) o ambas. Además los agregados silicoclásticos con vidrio, posiblemente procedieron de las tierras altas volcánicas, por ejemplo del volcán Chichón y de materiales lagunares o pantanos que contenían diatomeas.

La fuente de las pastas que contienen fósiles de diatomeas (ópalo) probablemente fue cercana a lagos o lagunas de agua dulce. Por otra parte, dado que el sílice le confiere estabilidad a las pastas cerámicas, es dable pensar que el uso de materiales que contenían estos fósiles, fue beneficioso.

\section{AGRADECIMIENTOS}

Se agradece al Dr. Rodrigo Liendo Stuardo, director responsable del proyecto Chinikihá, profesor Investigador Titular del Instituto de Investigaciones Antropológicas de la Universidad Nacional Autónoma de México, quién autorizó del uso de los datos cerámicos. Se agradece a Marcela Barver por la ayuda brindada. Agradecimientos al revisor del texto Dr. Guillermo Alvarado.

\section{REFERENCIAS BIBLIOGRÁFICAS}

CHUNG, H., 2009: La cronología de Chichén Itza.- 276 págs. Korean Studies Information Co, Ltd. Korea.

DEER, W.A., HOWIE, R.A. \& ZUSSMAN J., 1966: An Introduction to the RockForming Minerals.- 528 págs. Longman Group, US.

ERVAN, H. \& GARRISON, G., 1998: Geological methods for archaeology.- 343 págs. Oxford University Press, US.

DGG (1983): Carta Geológica, Tenosique E15-9. Escala 1:250 000, Dirección General de Geografía. Coordinación General de los Servicios Nacionales de Estadística, Geografía e Informática. Estados Unidos Mexicanos. 
FORD, A. \& SPERA, F., 2007: Fresh volcanic glass shards in the pottery sherds of the maya lowlands.- Research Reports in Belizean Archaeology, Inst. of Archaeol., NICH, Belize, 4:111-118.

FORD, A. \& ROSE, W.I., 1995: Volcanic ash in ancient Maya ceramics of the limestone lowlands:implications for prehistoric volcanic activity in the Guatemala Highlands.- J. Volcanol. and Geoth. Res. $66: 149-162$

INEGI (1983): Carta Geológica, Villahermosa E15-8.- Escala 1:250 000, Dirección General de Geografía. Estados Unidos Mexicanos.

JIMÉNEZ, S., 2007: Consumo, distribución y producción especializada de los bienes cerámicos durante el clásico tardío-Terminal de Chinikihá, Chiapas.- 25 págs. Univ. Nac. Autónoma de México, México D.F. [Inf. Interno].

JIMÉNEZ, S., 2009: Apuntes preliminares y catalogación de la cerámica de Chinikihá, Chiapas, México. Temporada de gabinete 2007-2009. Parte 1 y parte 2.- 125 págs. Univ. Nac. Autónoma de México, México D.F. [Inf. Interno].

LIENDO, R., 2007: Proyecto arqueológico Chinikihá, Temporada 2006. Informe de actividades.- 97 págs. Foundation for the Advancement of Mesoamerican Studies, Inc. (FANSI) [Inf. Interno].

LÓPEZ, S., 1991: La cerámica de Pomoná, Tabasco. Un ejemplo del clásico maya en las tierras bajas noroccidentales.- 201 págs. Univ. de las Américas, Puebla [Inf. Interno].

LÓPEZ, S., 2005: Dynamics of engagement in the Usumacinta River valley and the coastal plains of Tabasco: Traversing terminal classic hypotheses.- En: VARELA, S \& FOIAS, A. (eds): Geographies of
Power: Understanding the Nature of Terminal Classic Pottery in the Maya Lowlands.- BAR International Series, Oxford, 1447: 41-60

MACÍAS, J.L., 2005: Geología e historia eruptiva de algunos de los grandes volcanes activos de México.- Bol. Soc. Geol. Mexicana (Vol. Conmemorativo del Centenario, Temas Selectos de la Geología Mexicana), LVII(3): 379-424.

MUÑOZ, A., 2006: Power, Production and Prestige: Technological Change in the Late Classic Ceramics of Piedras Negras, Guatemala.- 404 págs. Univ. de Arizona [Tesis Ph.D.].

NOOREN, C.A.M., HOEK, W.Z., TEBBENS, L.A. \& DEL POZZO, A.L.M., 2008: Tephrochronological evidence for the late Holocene eruption history of El Chichón Volcano, Mexico.- Geof. Internacional, 48(1): 97-112.

RANDS, R., 1967: Ceramic Technology and Trade in the Palenque Region, Mexico.- En: RILEY, C. \& TAYLOR, W. (eds): Essays in Honor of Leslie Spier.- Southern Illinois University Press:137-150.

RANDS, R., 1974: The Ceramic Sequence at Palenque, Chiapas.- En: HAMMOND, N. (ed.): Mesoamerican Archaeology, New Approaches.- Univ. of Texas Press: 51-76.

RANDS, R., 1987: Ceramic patterns and traditions in the Palenque area.- En: RICE, P. \& SHARER, R. (eds): Maya ceramics: Papers from the 1985. Maya ceramic conference.- BAR International Series, 345(i): 203-238.

RANDS, R., 2007: Palenque and selected survey sites in Chiapas and Tabasco.- En: MARKEN, B.M. (ed.): Palenque recent 
Investigations at the Classic Maya Center.Alta Mira Press: 25-56.

RANDS, R. \& RANDS, B., 1957: The Ceramic Position of Palenque, Chiapas.- Amer. Antiquity, 23(2):140-150

SAN ROMÁN, E., 2005: El Clásico Temprano en Palenque a través de su cerámica.Lakamha'. Segunda época, 16: 8-9.

SHEPARD, A.O., 1964: Temper identification: "Technological Sherd-Splitting" or an unanswered challenge.- Amer. Antiquity, 29: 518-520.
SHEPARD, A.O., 1976: Ceramics for the Archaeologist.- 414 págs. Carnegie Institution.of Washington.

SIMMONS, M., P \&. BREM, G.F., 1979: The Analysis and Distribution of Volcanic Ash Tempered Pottery in the Lowland Maya.Amer. Antiquity, 44(1):79-91.

STUART, D. \& MORALES, A., 2003: Chinikiha: The Moderns Threat to an Acient Maya Kingdon.- http://www.mesoweb.com/reports/ chinikiha.html [Consulta: 10 de noviembre, de 2010]. 